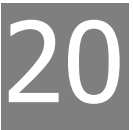

\title{
Contact Lens Care Solutions and Ocular Surface
}

dosó Manuel Gonzáloz Móijomg

\begin{abstract}
SUMMARY
Safe wear of non-daily disposable contact lenses require care systems to ensure disinfection and cleaning to allow the lens to be reused after a period of non lens wear (typically overnight). To do so, modern care systems, also known as multipurpose solutions (MPS) or multipurpose disinfecting solutions (MPDS) try to mimic the environment of the ocular surface regarding $\mathrm{pH}$, osmolality, surface tension or viscosity while introducing other singular properties as a wide-spectrum antimicrobial activity and high cleaning performance. This delicate equilibrium ex properties is challenging and sometimes results in adverse events that have drawn the attention of the CL industry and scientific community to these systems with an increased intensity during the past decade.

Even with disposable lenses, safety is still a concern and presently we are in front of a new paradigm of solutions development, which must provide reinforced disinfecting capabilities and at the same time improve the compatibility with the ocular surface. Indeed, disinfection efficacy against different new strains of microorganisms and compatibility with the ocular surface have been two aspects widely covered in the recent scientific literature, topics that will also be addressed in this chapter.
\end{abstract}

\section{INTRODUCTION}

Care solutions are essential for contact lens (CL) safety and durability. They were first intended for cleaning and disinfecting lenses for non-planned

Clinical \& Experimental Optometry Research Lab (CEORLab), University of Minho, Campus de Gualtar 4710-057, Braga-Portugal; E-mail: jgmeijome@fisica.uminho.pt 
replacement lenses, commonly using products with poor compatibility with the ocular surface, such as hydrogen peroxide, chlorhexidine or benzalconium chloride. In spite of their efficacy against microorganisms, they were somewhat inconvenient, at least in their earlier formulations particularly when used with hydrophilic lenses that absorb such components into their matrix.

Care systems were initially complex in the sense that the wearer must use different products; nowadays the care systems have evolved into complex formulations that meet all the requirements for a care system in a single package in order to make its use easier and more convenient to the CL wearer and improve compliance. However, this combination of properties into the same package requires an optimal balance between the disinfecting and cleaning activity and the respect for the ocular surface homeostasis.

Today, development of effective care systems is a challenging task once they have to be effective for cleaning and disinfection, and at the same time respect the physiology of the ocular surface, be convenient for the wearer and when possible and improve the comfort of the CL during the whole day. These are the requirements for current MPDS that represent over $80 \%$ of the all care systems prescribed (Morgan et al. 2010). With the advent of disposable CL cleaning capability has become less important and industry makes efforts to provide MPDS with new features that address one of the main unsolved issues for contact lens wearers: all day comfortable wear. As a result, current MPDS are the result of complex chemical and biological engineering that must have a critical combination of properties such as $\mathrm{pH}$, osmolality, viscosity maintaining high standards of disinfection and cleaning efficacy.

\section{REQUIREMENTS AND COMPOSITION OF CURRENT CARE SOLUTIONS}

The basic requirements for a CL care system are to clean tear and environmental products deposited every day on the surface of the lens, to remove the materials that had been strongly adhered to the CL material, to disinfect the lens by reducing the populations of microorganisms to safety levels, to warrant that the CL material remains fully hydrated, and desirably, to improve the CL wetting properties to extend wearing comfort during the whole day-of wearing.

Table 1 shows some examples of soft CL MPS marketed and their composition. Composition of MPDS is critical because of the potential impact on the interaction with the contact lenses and with the ocular surface. Similar compositions can be described for rigid gas permeable lenses (RGP). Eventually, in the case of RGP materials, due to their inability to absorb the active ingredients, other preservative or biocidal agents as derivates of 


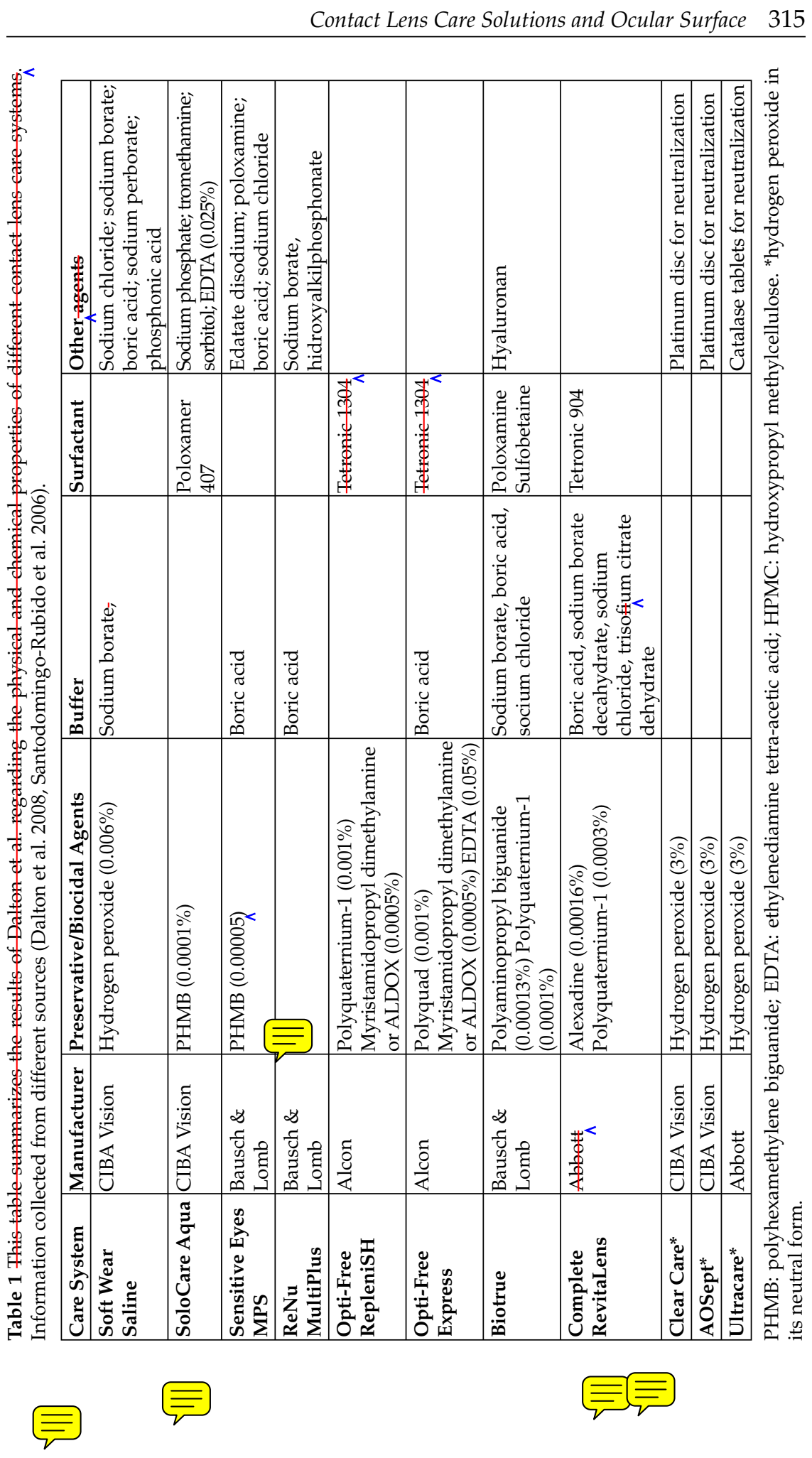


chlorhexidine or even benzalkonium chloride (BAK), no longer incorporated to soft CL care systems, can be used. Other major difference with soft CL systems is that such MPDS use to have higher viscosity to improve the comfort of the CL by increasing the wetting capabilities of the surface and cushioning the contact between the RGP CL and the ocular surface.

\section{PROPERTIES OF CARE SYSTEMS}

Considering the intimal relationship of care solutions with the contact lens material and the ocular surface, the properties of the care systems are critical for a good performance.

Some of the most relevant properties include: $\mathrm{pH}$, osmolality, surface tension, and viscosity. The relevance of these properties, from the clinical point of view range from the impact on insertion comfort to the dynamic interaction of the lens with the ocular surface (cornea, bulbar conjunctiva and tarsal conjunctiva). In brief, a solution with $\mathrm{pH}$ or osmolality out of the physiological range (Table 2), could result in discomfort at insertion; an excessive surface tension will result in decreased spread of the solution over the CL or the ocular surface; too low viscosity will decrease the lubricating effect of the tears in the interaction of the lens with the ocular surface; too high viscosity will result in an improved lubricating effect but eventually could result in poor visual due to irregularities in the tear film or even lens binding to the surface. Dalton et al. evaluated these properties in 10 care systems including 7 MPS solutions and 3 neutralized hydrogen peroxide solutions (Dalton et al. 2008). The main results of this study are summarized in Table 2 along with the normal range for the normal tears.

Although the normal $\mathrm{pH}$ of tears has been defined between 6.2 and 9.0, care systems must be formulated to maintain the $\mathrm{pH}$ between 6.6 and 7.8, which is also known as the comfort zone (Carney and Hill 1976). As seen in table 2, most of the solutions do respect these desired limits, although presenting significant differences among them.

Beyond their intrinsic properties, it is also interesting to evaluate the potential changes of such parameters overtime. Previous studies have demonstrated that properties such as $\mathrm{pH}$ can remain within the normal physiological range during a significant period of time in spite of some fluctuations (Lopez-Alemany and Montes 1998). Recent studies have addressed the changes in $\mathrm{pH}$ and osmolality over a month for different products and particularly when the lens cases are subjected to different cleaning, rinsing and drying procedures (Abengozar-Vela et al. 2011). Some cleaning and drying procedures of the storage lens cases can affect properties such as-osmolality (Abengozar-Vela et al. 2011) such that air-drying, today recommended by several manufacturers, can increase significantly the 
Table 2 This table summarizes the results of Dalton et al. regarding the physical and chemical properties of different contact lens care systems (Dalton et al. 2008).

\begin{tabular}{|l|c|c|c|c|}
\hline Care System & $\mathbf{p H}$ & $\begin{array}{c}\text { Osmolality } \\
(\mathbf{m O s m} / \mathbf{K g})\end{array}$ & $\begin{array}{c}\text { Surface Tension } \\
(\mathbf{m N} / \mathbf{m})\end{array}$ & $\begin{array}{c}\text { Viscosity } \\
\left(\mathbf{c P} \text { @ 34 } \mathbf{~}^{\mathbf{C}} \mathbf{)}\right.\end{array}$ \\
\hline Average for Fears & $6.2-9.0$ & 305 & $40-46$ & $1.5-5.0$ \\
\hline Soft Wear Saline & 6.97 & 303.5 & 67.9 & 0.70 \\
\hline Solo Care Aqua & 7.23 & 310 & 35.1 & 0.81 \\
\hline Sensitive Eyes MPS & 7.29 & 286.7 & 38.0 & 0.83 \\
\hline ReNu MultiPlus & 7.38 & 286.2 & 36.3 & 0.84 \\
\hline Complete Moisture Plus & 7.28 & 304.3 & 40.5 & 1.92 \\
\hline Opti-Free RepleniSH $^{\prime}$ & 7.88 & 277.2 & 29.7 & 0.71 \\
\hline Opti-Free Express $^{*}$ & 7.82 & 225.0 & 31.2 & 0.76 \\
\hline Clear Care* $^{*}$ & 6.76 & 293.7 & 42.9 & 0.69 \\
\hline AOSept $^{*}$ & 6.66 & 290.7 & 70.3 & 0.69 \\
\hline Ultracare* $^{*}$ & 7.18 & 329.0 & 43.2 & 0.86 \\
\hline
\end{tabular}

*hydrogen peroxide in its neutral form.

osmolality of the solution where the lenses are immersed overnight if a tissue-wiping step is not added to the process.

\section{DISINFECTING EFFICACY}

Disinfecting efficacy of CL care systems is another critical property and it is measured as the reduction of viable organisms or colony forming units (CFU's) during the recommended disinfection time or during a certain period of time as set by FDA and ISO standards. The usual number of organisms inoculated is in the order of $10^{6}$ units or CFU's. Typically, a care system should be challenged against several organisms, including gram-negative and gram-positive bacteria, mold and yeast. The results are expressed in terms of logarithm units of reduction such that $1 \log$ unit corresponds to the elimination or neutralization of $90 \%$ of the organisms initially inoculated ( $10^{5}$ CFU's remaining); similarly 2 log units are equivalent to a double reduction of $90 \%$ ( $10^{4} \mathrm{CFU}$ 's remaining), etc. After a 5 log reduction, only 10 CFU's will remain and after a 6 log reduction, only 1 CFU will remain. Standards require at least a $3 \log$ reduction for bacteria (gram-positive and gram-negative) and $1 \log$ reduction for mold and yeast.

Figure 1 represents the requirements set by FDA and ISO required for a given care system although these regulations are under review to incorporate more microorganisms.

Nowadays most CL care systems and particularly those new systems being developed are being challenged against a much wider spectrum of microorganisms, including those responsible for serious public health concerns as antibiotic-resistance microorganisms and clinical isolates 


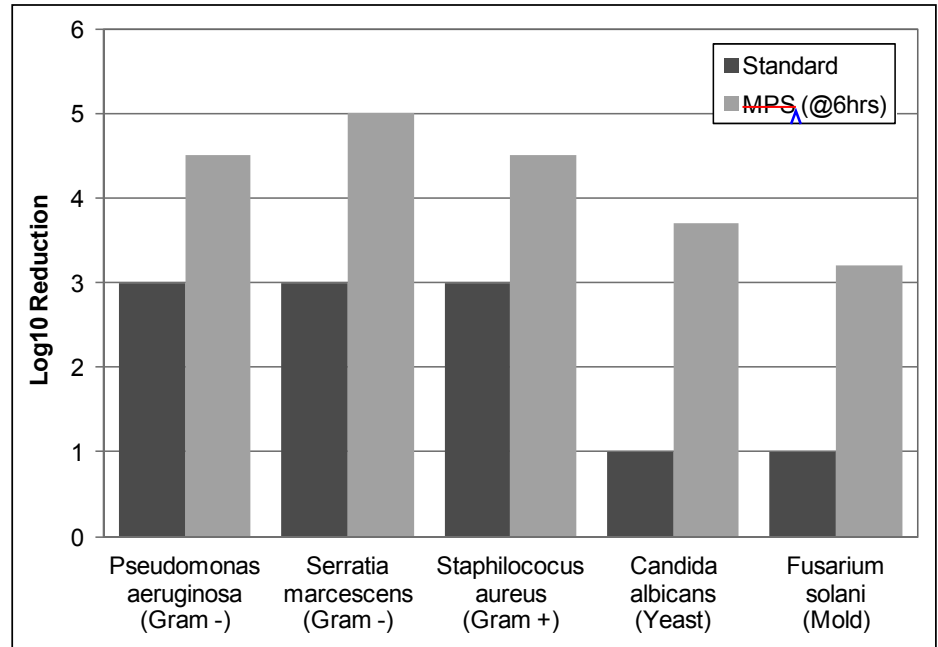

Figure 1 Standards of reduction in CFU viability and performance of a given MPDS against gram-positive and gram-negative bacteria, yeast and mold $_{\bar{\kappa}}$

(extracted from contaminated cases or infected eyes instead of using only strains from laboratory cultures) in order to provide stronger evidences of the efficacy of the MPDS against "real world microorganisms". This has been the case in a study published by Zhu et al. (Zhu et al. 2007) where up to 10 gram-positive bacteria, 8 gram-negative bacteria and 3 fungi ( 2 yeast and 1 mold) were evaluated. Other study conducted by Kilvington et al. (Kilvington et al. 2010) on a new MPDS also evaluated differ microorganisms frem ${ }_{\lambda}$ those recommended by FDA and ISO standards.

Acanthamoeba castellanii, one of the most virulent and challenging ocular microorganism, either in the trophozoite and cyst forms were evaluated, providing quite different results among different solutions, particularly in the cystic form where some solutions were ineffective to achieve a 3 $\log$ reduction; such results reinforce the need for tighter standards for MPDS evaluation. Further, these authors evaluated the efficacy of different solutions after evaporation of the solution, a condition that could derive from poor compliance from the patient and that could act as a risk factor for ocular infection due to reduced biocidal activity of the solution and/or to increased resistance of the microorganisms in such environment.

\section{INTERACTIONS OF CARE SYSTEMS WITH THE OCULAR SURFACE}

The components of the CL solutions can be related to several adverse reactions in the ocular surface by mediating a toxic reaction due to prolonged 
contact once the products adhered to the lens material leak to the ocular surface. Several studies have tried to relate these clinical events presenting in the form of ocular surface damage to the presence of certain components in the care systems. The disinfecting or biocidal agents or their interaction with other components included in the formulation have been pointed as responsible for such reactions.

Polyhexamethylene biguanide (PHMB) also known as polyhexanide, Dymed, polyhexidine and polyaminopropyl biguanide has been frequently related to solution induced corneal staining (SICS). However, the laboratory research was unable to establish an in vitro relationship with the in vivo clinical findings. On the other hand, polyquaternium-1 also known, as Polyquad is other frequently used disinfecting agent also involved in such laboratory tests whose results are described in further detail below and will be summarized in Table 3.

A study from Santodomingo-Rubido et al. ebserved that different concentrations of certain ingredients commonly found in commercial CL solutions could be related with higher or lower citotoxicity of experimental cell lines from animal models (mouse lung cells) (Santodomingo-Rubido et al. 2006). Interestingly, the authors found that while some MPDS solutions were invariably associated to high or low levels of citotoxicity at any concentration between 1.25 to $10 \%$ of MPDS, the citotoxicity activity was largely dependent on concentration for other MPDS which was attributed to the biocidal agent included or to the combination of this agent with other ingredients such as buffer ingredients. Was also interesting to observe how certain surfactants such as Poloxamer 237 or Poloxamer 407 were invariably related to low and high citotoxicity irrespective of the concentration used, while citotoxicity of other surfactant Tetronic 1107 was dependent on concentration. Finally, cytotoxicity of most isotonic and buffer agents was found to be highly dependent on concentration (Santodomingo-Rubido et al. 2006).

Other authors used to look at these issues in different ways using different laboratory assays. Chuang et al. used ApopTag Fluorescein Apoptosis assay to detect cell death after exposure to different MPDS, fluorescein permeability to investigate the corneal epithelial barrier function and tight junction proteins zonula occludens (ZO)-1 and occludin were evaluated using immunofluorescent staining (Chuang et al. 2008). Imayasu et al also evaluated the tight junction integrity of the corneal epithelial cells with ZO-1 (tight junction-related protein) labeling under laser confocal microscopy. 
Table 3 This table summarizes the results of different studies addressing the citotoxicity of several MPDS.

\begin{tabular}{|l|l|l|}
\hline Authors (year) & Solutions evaluated & Main outcomes \\
\hline $\begin{array}{l}\text { (Santodomingo- } \\
\text { Rubido et al.2006) }\end{array}$ & $\begin{array}{l}\text { Complete Moisture Plus* } \\
\text { MeniCare Soft } \\
\text { Opti-Free Express } \\
\text { ReNu Multiplus } \\
\text { Renu MoistureLoc* } \\
\text { SoloCare Aqua }\end{array}$ & $\begin{array}{l}\text { Citotoxicity Activity (at medium } \\
\text { concentration of 5\%) } \\
\text { Complete }<\text { MeniCare }<\text { SoloCare } \\
<\text { OptiFree }<\text { ReNu MultiPlus }= \\
\text { ReNu MoistureLoc }\end{array}$ \\
\hline (Chuang et al.2008) & $\begin{array}{l}\text { Complete Easy Rub } \\
\text { OptiFree Express } \\
\text { OptiFree RepleniSH } \\
\text { ReNu Multiplus }\end{array}$ & $\begin{array}{l}\text { Apoptosis Rates } \\
\text { Complete }<\text { ReNu < OptiFree } \\
\text { RepleniSH < OptiFree Express }\end{array}$ \\
\hline (Imayasu et al.2008) & $\begin{array}{l}\text { MPS1: PHMB } \\
\text { MPS2: PHMB + Poloxamine + } \\
\text { Boric Acid } \\
\text { MPS3: Alexidine + Poloxamine } \\
+ \text { Boric Acid } \\
\text { MPS4: Polyquad + Poloxamine } \\
+ \text { Boric Acid }\end{array}$ & $\begin{array}{l}\text { Transepithelial Electrical Resistance } \\
\text { Culture in Collagen Membrane } \\
\text { @120 min.) } \\
\text { MPS1 < MPS3 < MPS2 < MPS4 } \\
\text { Combination of poloxamine and } \\
\text { boric acid as potential cause of tigh } \\
\text { junction damage }\end{array}$ \\
\hline $\begin{array}{l}\text { (Mowrey-McKee et } \\
\text { al.2002) }\end{array}$ & $\begin{array}{l}\text { AOSept (neutral) } \\
\text { Complete Comfort Plus } \\
\text { OptiFree Express } \\
\text { ReNu } \\
\text { ReNu Multiplus } \\
\text { SoloCare Soft }\end{array}$ & $\begin{array}{l}\text { Different Assays } \\
\text { Only OptiFree Express was } \\
\text { considered citotoxic for trypan } \\
\text { blue uptake test and cell regrowth } \\
\text { (absent) following exposure to test } \\
\text { material. ReNu products showed } \\
\text { citotoxicity on quantification of } \\
\text { viable cells }\end{array}$ \\
\hline
\end{tabular}

* Currently not marketed.

To investigate the changes of ultrastructure in tight junctions of human corneal epithelial cells, the same authors observed an ultrathin cross-section of the cell on collagen membrane using transmission electron microscopy. (Imayasu et al. 2008) Mowrey-McKee et al., evaluated citotoxicity of CL care systems using different modifications of the United States Pharmacopeia (USP) elution test required by U.S. Food and Drug Administration (FDA) and global standards organizations such as the International Standards Organization (ISO) for in vitro cytotoxicity testing of lens care solutions (Mowrey-McKee et al. 2002). Results of these and other evaluations are summarized in table 3. Despite all these studies found significant differences between the citotoxicity of different MPS solutions, the different experimental conditions and techniques used limit the ability to compare and summarize the results from all these studies. Moreover, their results obtained "in vitro" did not relate much with the clinical observations such that, care systems reported as being safe in the clinical setting revealed worse behaviour in the laboratory assays and vice-versa. 
CHALLENGES FOR CURRENT MPDS DEVELOPMENT

After the outbreaks of MPDS-related infections during the last 5 years (Khor et al. 2006, Patel and Hammersmith 2008, Tu and Joslin 2010), there has been a great deal of discussion regarding the efficacy of these systems and the sufficiency of current FDA and ISO regulations regarding the tests that new systems must undergo for approval.

This issue is aggravated by the known lack of compliance of many CL wearers with the correct guidelines of use of their CL and care systems. Such behaviours could eventually result in an increased risk for most of these wearers. Among some of the modifiable patient compliance-related behaviours, the following have been identified as increased risk factors (relative risk, RR) for CL associated infections: inadequate hand-washing $(\mathrm{RR}=4.5$ (Stapleton et al. 2007)), inadequate case cleaning $(\mathrm{R} R=4.0$ (Houang et al. 2001)), failure to use correct disinfecting solution ( $R R=21.8$ to 55.9; (Houang et al. 2001, Radford et al. 1998), failure to rub and rinse lenses $(\mathrm{RR}=3.5$ (Radford et al. 1995)) and topping off solution ( $R R=2.5$ (Saw et al. 2007)), among others (Morgan et al. 2011).

As a result, some new multipurpose and disinfecting solutions have been developed to meet criteria beyond the current regulatory standards. As such, new organisms as different strains of bacteria (i.e. metacilyn resistance Staphylococcus aureus also known as MRSA) or amoeba such as Acanthamoeba either in its trophozoite or cystic forms have became usual in the evaluation of biocidal activity of new care systems as previously described in this chapter (Kilvington et al. 2010; Zhu et al. 2007).

\section{ROLE OF CARE SOLUTIONS IN IMPROVING CL'S CLINICAL PERFORMANCE}

When it comes to the relationship between adverse events in the ocular surface and the potential impact of MPS on their aetiology, it is hard to establish a direct relationship. However, it is commonly accepted that the interaction between some MPDS ingredients and the ocular surface, mediated or not thought particular interactions of those with the CL material, can have a negative impact in the tolerance of CL with the ocular surface (Andrasko et al. 2006, Carnt et al. 2007, Garofalo et al. 2005).

Nowadays, care systems no longer pretend only to perform well in terms of cleaning and disinfecting efficacy but play also an important role in CL performance by improving physical comfort at insertion and through the whole period of wear.

Indeed, several studies pointed out the role of care systems in preventing or recovering from ocular surface damage related to CL as is the case of superficial punctuate corneal staining er $_{\Lambda}$ SICS. SICS identification required 
that at least 4 out of 5 regions of the cornea are affected by this superficial staining (Fig. 2).

Current knowledge advocates for the role of care systems in reducing SICS events. Hydrogen peroxide has been proposed as an effective strategy in case of corneal staining potentially related to multipurpose solution (Carnt et al. 2007, Papas et al. 2007). Additionally, the pre-application of carboxymethylcellulose (CMC) lubricating and wetting drops (artificial tears) has demonstrated to significantly reduce the incidence of corneal staining in conventional hydrogel CL (Coles et al. 2004) and silicone hydrogel soft CL wearers (Paugh et al. 2007).

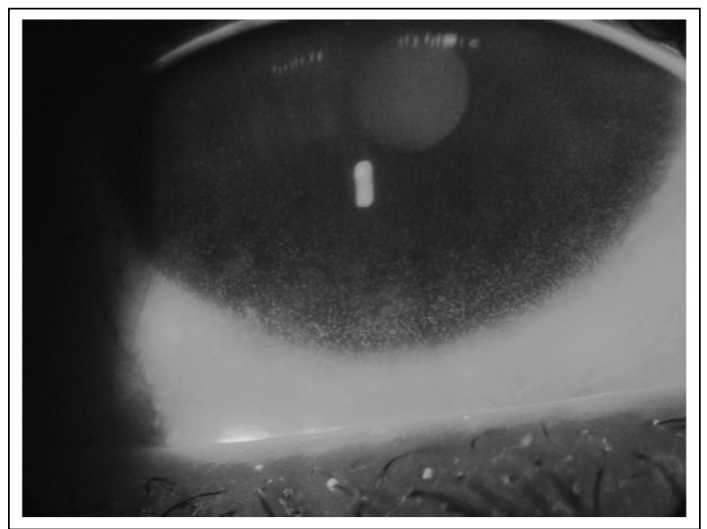

Figure 2 Typical appearance of a peripheral corneal staining covering 4 quadrants (superior one not seen in this photograph).

Color image of this figure appears in the color plate section at the end of the book.

Another field of interest is the introduction of wetting agents and other active ingredients into new MPDS formulations to improve the hydration retention of CL materials and improve the long-term comfort. With this purpose, new solutions such as Biotrue (Bausch \& Lomb) incorporate derivates of hyaluronic acid to make the lens material hydration to last and the company claims up to 20 hours of hyaluronan retention in the matrix of different hydrogel and silicone hydrogel CL with the potential benefit of improved end-of-day comfort (Scheuer et al. 2010). An additional purpose of this formulation is to maintain native tear proteins in their natural state instead of denaturating onto the CL material, providing a chance to develop adverse reactions on the ocular surface (Barniak et al. 2010). Although the benefits of these new formulations on lens dehydration and whole day lens comfort will need to be further demonstrated by independent studies, the active implication of the CL care systems to improve CL comfort over time present as a promissory strategy to improve the whole CL wearing experience and eventually decrease the drop-out rate among CL wearers 
as most of these discontinuations seem to be directly related with dryness and discomfort symptoms (Pritchard et al. 1999).

\section{CONCLUSIONS}

Finally, the modes in which care systems are used seem to be critical to improve their performance in several aspects. Although in the recent past MPDS use to include a non-rub claim, it has been proved that the inclusion of rub and rinse steps improves significantly the performance of MPDS solutions in terms of disinfection efficacy (Zhu et al. 2011). Rub and rinse step in addition to other compliance recommendations such as tissue-wipe and air-dry demonstrated to be very effective in eliminating microorganisms an $\equiv$ pilms from CL storage cases (Wu et al. 2010, Wu et al. 2011).

$\overline{7}$ hese strategies, in addition to the high antimicrobial efficacy of modern MPDS formulations (some of them at the expense of including a double biocidal agent in their formulation) should render positive results in terms of reduced CL case contamination thus lowering the incidence of adverse events related to contact lens wear and their potential interaction with care systems ${ }_{\lambda}$

\section{DISCLOSURE}

The author is or has been during the last 5 years consultant for several companies with interests in contact lens care systems. The author does not have any financial interest in those care systems or brands.

\section{REFERENCES}

Abengozar-Vela, A., F.J. Pinto, J.M. Gonzalez-Meijome, M. Rallo, C. Seres, M. Calonge and M.J. Gonzalez-Garcia. 2011. Contact Lens Case Cleaning Procedures Affect Storage Solution pH and Osmolality. Optom. Vis. Sci. 88: 1414-21.

Andrasko, G.J., K.A. Ryen, R.J. Garofalo and J.M. Lemp. 2006. Compatibility of Silicone Hydrogel Lenses With Multi-Purpose Solutions. ARVO Meeting Abstracts 47: 2392.

Barniak, V.L., S.E. Burke and S. Venkatesh. 2010. Comparative evaluation of multi-purpose solutions in the stabilization of tear lysozyme. Cont. Lens Anterior. Eye 33: S7-11.

Carney, L.G. and R.M. Hill. 1976. Human tear pH. Diurnal variations. Arch. Ophthalmol. 94: 821-824.

Carnt, N.A., M.D.P. Willcox, V.E. Evans, T.J. Naduvilath, D. Tilia, E.B. Papas, D.F. Sweeney and B.A. Holden. 2007. Corneal staining with various contact lens solution-silicone hydrogel lens combinations and its significance: The IER Matrix Study. Contact Lens Spectrum 22: 38-43.

Chuang, E.Y., D.Q. Li, F. Bian, X. Zheng and S.C. Pflugfelder. 2008. Effects of contact lens multipurpose solutions on human corneal epithelial survival and barrier function. Eye Contact Lens 34: 281-286. 
Coles, M.L., N.A. Brennan, V. Shuley, J. Woods, C. Prior, J.G. Vehige and P.A. Simmons. 2004 The influence of lens conditioning on signs and symptoms with new hydrogel contact lenses. Clin. Exp. Optom. 87: 367-371.

Dalton, K., L.N. Subbaraman, R. Rogers and L. Jones. 2008. Physical properties of soft contact lens solutions. Optom. Vis. Sci. 85: 122-128.

Garofalo, R. J., N. Dassanayake, C. Carey, J. Stein, R. Stone and R. David. 2005. Corneal staining and subjective symptoms with multipurpose solutions as a function of time. Eye Contact Lens 31: 166-174.

Houang, E., D. Lam, D. Fan and D. Seal. 2001. Microbial keratitis in Hong Kong: relationship to climate, environment and contact-lens disinfection. Trans. R. Soc. Trop. Med. Hyg. 95: 361-367.

Imayasu, M., A. Shiraishi, Y. Ohashi, S. Shimada and H.D. Cavanagh. 2008. Effects of multipurpose solutions on corneal epithelial tight junctions. Eye Contact Lens 34: 50-55.

Khor, W. B., T. Aung, S.M. Saw, T.Y. Wong, P.A. Tambyah, A.L. Tan, R. Beuerman, L. Lim, W.K. Chan, W.J. Heng, J. Lim, R.S. Loh, S.B. Lee and D.T. Tan. 2006. An outbreak of Fusarium keratitis associated with contact lens wear in Singapore. JAMA 295: 2867-2873.

Kilvington, S., L. Huang, E. Kao and C.H. Powell. 2010. Development of a new contact lens multipurpose solution: Comparative analysis of microbiological, biological and clinical performance. J. Optom. 3: 134-142.

Lopez-Alemany, A. and I Montes Mico. 1998. $\mathrm{pH}$ of multipurpose contact lens solutions over time. Cont.Lens Anterior Eye 21: 7-10.

Morgan, P. B., C.A. Woods, I.G. Tranoudis, M. Helland, N. Efron, R. Knajian, C.N. Grupcheva, D. Jones, K.-O. Tan, A. Pesinova, O. Ravn, J. Santodomingo, E. Vodniaznszky, N. Erdinest, H.I. Hreinsson, G. Montani, M. Itol, J. Bendoriene, E. van der Worp, J. Hsiano, G. Phillips, J.M. Gonzalez-Méijome, S. Radu, V.Belousov and J.J. Nichols. 2010. International contact lens prescribing in 2009. Contact Lens Spectrum 30-36.

Morgan, P.B., N.Efron, H. Toshida and J.J. Nichols. 2011. An international analysis of contact lens compliance. Cont. Lens Anterior. Eye 34: 223-228.

Mowrey-McKee, M., A. Sills and A. Wright. 2002. Comparative cytotoxicity potential of soft contact lens care regimens. CLAO J. 28: 160-164.

Papas, E.B., N. Carnt, M.D. Willcox and B.A. Holden. 2007. Complications associated with care product use during silicone daily wear of hydrogel contact lens. Eye Contact Lens 33: 392-393.

Patel, A. and K. Hammersmith. 2008. Contact lens-related microbial keratitis: recent outbreaks. Curr. Opin. Ophthalmol 19: 302-306.

Paugh, J.R., H.J. Marsden, T.B. Edrington, P.N. Deland, P.A. Simmons and J.G. Vehige. 2007. A pre-application drop containing carboxymethylcellulose can reduce multipurpose solution-induced corneal staining. Optom.Vis. Sci. 84: 65-71.

Pritchard, N., D. Fonn and D. Brazeau. 1999. Discontinuation of contact lens wear: a survey. Int. Contact Lens Clin. 26: 157-162.

Radford, C.F., A.S.Bacon, J.K. Dart and D.C. Minassian. 1995. Risk factors for acanthamoeba keratitis in contact lens users: a case-control study. BMJ 310: 1567-1570.

Radford, C.F., D.C. Minassian and J.K. Dart. 1998. Disposable contact lens use as a risk factor for microbial keratitis. Br. J. Ophthalmol. 82: 1272-1275.

Santodomingo-Rubido, J., O. Mori and S.Kawaminami. 2006. Cytotoxicity and antimicrobial activity of six multipurpose soft contact lens disinfecting solutions. Ophthalmic Physiol. Opt. 26: 476-482.

Saw, S. M., P.L. Ooi, D.T. Tan, W.B. Khor, C.W. Fong, J. Lim, H.Y. Cajucom-Uy, D. Heng, S.K Chew, T. Aung, A.L. Tan, C.L. Chan, S. Ting, P.A. Tambyah and T.Y. Wong. 2007. Risk factors for contact lens-related fusarium keratitis: a case-control study in Singapore. Arch. Ophthalmol. 125: 611-617. 
Scheuer, C.A., K.M. Fridman, V.L. Barniak, S.E. Burke and S. Venkatesh. 2010. Retention of conditioning agent hyaluronan on hydrogel contact lenses. Cont. Lens Anterior. Eye 33: S2-S6.

Stapleton, F., L. Keay, I. Jalbert and N. Cole. 2007. The epidemiology of contact lens related infiltrates. Optom. Vis. Sci. 84: 257-272.

Tu, E.Y. and C.E. Joslin. 2010. Recent outbreaks of atypical contact lens-related keratitis: what have we learned? Am. J. Ophthalmol. 150: 602-608.

Wu, Y.T., H. Zhu, M. Willcox and F. Stapleton. 2010. Impact of air-drying lens cases in various locations and positions. Optom. Vis. Sci. 87: 465-468.

Wu, Y.T., H. Zhu, M. Willcox and F. Stapleton. 2011. The effectiveness of various cleaning regimens and current guidelines in contact lens case biofilm removal. Invest Ophthalmol. Vis. Sci. 52: 5287-5292.

Zhu, H., A. Ding, M. Bandara, M.D. Willcox and F. Stapleton. 2007. Broad spectrum of antibacterial activity of a new multipurpose disinfecting solution. Eye Contact Lens 33: 278-283.

Zhu, H., M.B. Bandara, A.K. Vijay, S. Masoudi, D. Wu and M.D. Willcox. 2011. Importance of rub and rinse in use of multipurpose contact lens solution. Optom. Vis. Sci. 88: 967-972. 